\title{
Dresden: Treffpunkt für alle Gesundheitsberufe
}

— Obwohl in Deutschland zahlreiche Fachkongresse sowohl für Ärzte als auch für professionell Pflegende und andere Gesundheitsberufe ausgerichtet werden, gibt es kaum Angebote, die berufsgruppenübergreifend den Patienten in den Fokus stellen. Hier ist die Gesundheitsbranche gefordert, ein professionelles Zusammenwirken aller beteiligten Berufe zu garantieren. Der Interprofessionelle Gesundheitskongress greift diesen Gedanken mit seinem Leitspruch "Viele Professionen - ein Patient" auf und lädt am 28. und 29. April 2017 erneut alle Gesundheitsberufe zum fachlichen Austausch ins Internationale Congress Center nach Dresden ein. "Sowohl der große Zuspruch beim letzten Kongress als auch die zahlreichen an uns herangetragenen Themenwünsche zeigen, wie wichtig dieser Austausch ist - und wie er zukünftig auch immer wichtiger wird", erklärt Falk $\mathrm{H}$. Miekley, Director Professional Care des Springer Medizin Verlages.

An den beiden Kongresstagen werden sich die rund 800 Kongressteilnehmer aus allen Gesundheitsberufen u.a. zu folgenden Schwerpunktthemen fortbilden können: Versorgung von Menschen mit Demenz im Akutkrankenhaus; bessere Kommunikation für eine bessere Gesundheitsversorgung;

\section{Charity for Charité}

\section{Spendengala zugunsten kleiner Patienten}

— Auf der Benefizgala Charity for Charité der Berliner Charité wurden am 24. November 2016 rund 260.000 Euro für schwerkranke Kinder gesammelt. Der Erlös der Spendengala kommt dem Aufbau eines Centrums zugute, in dem die Vision einer familiengerechten und medizinisch interdisziplinäreren Versorgung für schwer kranke Kinder unter einem Dach verwirklicht wer- den soll. „Mit dem Aufbau eines neuen Centrums will die Charité den kleinen Patienten ein Stück Zuhause in der Klinik bieten, z.B. durch integrierte Eltern-Kind-Zimmer", sagt Prof. Dr. Uwe Reuter, Medizinischer Leiter des CharitéCentrums 17 für Frauen-, Kinder- und Jugendmedizin.

www.charite.de

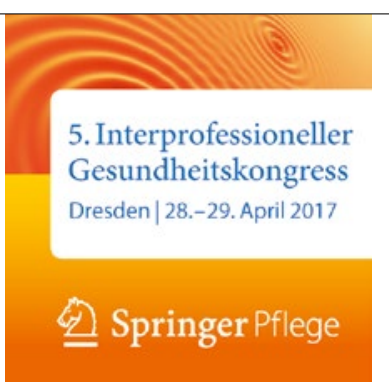

Ethik im Gesundheitswesen; gelungenes Prozessmanagement.

Zusätzlich zum interprofessionellen Programm wird es für die einzelnen Berufsgruppen wie gewohnt Angebote in den Modulen Pflege Spezial, Medizin Spezial und MFA Spezial geben. Auch in diesem Jahr finden wieder das HeilberufeSCIENCESymposium mit Posterpräsentation und ein Schülertag von Auszubildenden für Auszubildende statt.

www.gesundheitskongresse.de

\section{Defizite in Heimen \\ Augenärztliche Versorgung}

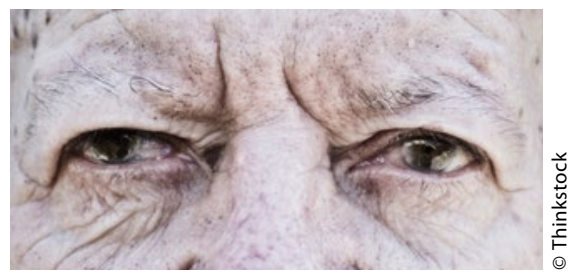

— Die augenärztliche Versorgung kommt bei Menschen in Pflege- und Seniorenheimen oft nicht an. Zu diesem Ergebnis kommt die Studie OVIS der Stiftung Auge. So gab rund die Hälfte der Studienteilnehmer in deutschlandweit 32 Heimen an, unter Sehproblemen zu leiden. Gleichwohl lag der letzte Augenarztbesuch durchschnittlich vier Jahre zurück. Rund die Hälfte gab dabei den Transport als größte Hürde an.

Zu den häufigsten festgestellten Erkrankungen in der Studie zählten grauer Star, altersabhängige Makuladegeneration und grüner Star. "Nicht selten fehlte es schlicht an einer passenden Brille für die Nähe und die Ferne", heißt es. Die Deutsche Ophthalmologische Gesellschaft rechnet damit, dass die Zahl altersbedingter Augenleiden bis 2030 um 20 bis 30\% zunehmen wird. Die Zahl der Behandlungsfälle bei den über 60-Jährigen könnte sogar um 35,8\% steigen.

www.stiftung-auge.de 